\title{
Dynamo effects in gravity
}

\author{
Boris Shevtsov ${ }^{1, \star}$ \\ ${ }^{1}$ Institute of Cosmophysical Research and Radio Waves Propagation FEB RAS, Paratunka, Kamchatka \\ region, Russia
}

\begin{abstract}
Nonlinear oscillations in the dynamic system of gravitational and material fields are considered. The problems of singularities and caustics in gravity, expansion and baryon asymmetry of the Universe, wave prohibition of collapse into black holes, and failure of the Big Bang concept are discussed. It is assumed that the effects of the expansion of the Universe are coupling with the reverse collapse of dark matter. This hypothesis is used to substantiate the vortex and fractal structures in the distribution of matter. A system of equations is proposed for describing turbulent and fluctuation processes in gravitational and material fields. Estimates of the diffusion parameters of such a system are made in comparison with the gravitational constant.
\end{abstract}

\section{Introduction}

From the turbulence and self-similarity of the wave motions of matter, it follows similar properties of space and the dynamic system of fields, which are considered in the theory of gravity. It is possible that nonlinear gravitational oscillations are similar to wave processes in a magnetic Dynamo system, in which the effects of field generation and their oscillations take place. What these effects mean for the fields of matter and gravity, and how they relate to the laws of conservation, must be understood. It is obvious that in the considered dynamic system there are interactions between scales, and the behavior of matter in the fields of gravity can be similar to the motion of a conservative impurity only in the first approximation.

In strong gravitational fields, one can expect deeply non-conservative and very destructive processes, such as the birth of a supernova, and this will require new assumptions about the properties of the wave motions of matter and physical space (gravity), the consequence of which may be the failure of the concepts of the Big Bang and Black holes, which developed as a tribute to the era of nuclear physics and General relativity. But these are other times that are marked by the successes of nonlinear dynamics and fractal theory, and in the spirit of this time, instead of dramatic singularities, we can propose the idea of inversion of matter from the visible state to the invisible in the strong gravitational fields of old massive stars in the centers of galaxies, and the return of dark matter and energy to the periphery of galaxies to form new stars. The idea of a cyclonic cycle, of course, is not new, but the justification for it is not enough. New models of gravity are needed, one of the possible models will be considered in this paper.

Fractality and scaling of wave motions are generated by the ratio of additive and multiplicative effects on the wave process, but what are the relations between the scales, and how stable they are,

\footnotetext{
^Corresponding author: bshev@ikir.ru
} 
it is still necessary to determine. In the study of complex natural systems it is necessary to consider the collective vibrations of the system, and not the behavior of its individual components. One approach for generating fractal wave motions, which is essentially a generalization of Brownian motion, lognormal process, and stochastic oscillator, will be proposed below.

It is not completely clear how the different kinds of physical interactions and gravity relate to each other. They are all different representations of the space connectivity. Below we will consider some examples of such representations.

To answer the question, what is common in the properties of wave motions at different scales, let us consider one of the scenarios of turbulence through the development of instability and growth of the dimension of wave motions, as an indicator of their complexity. For the description of wave motions we will use systems of Hypercomplex numbers [1].

\section{Wave forms}

We define a system of waves $\Psi(x)$ and $\Phi(x)$ by a pair of hypercomplex numbers, and define their change in differential forms:

$$
\left[i \partial^{v}+\gamma^{v}(x)\right] \Psi(x)=Y^{v}(x), \quad\left[i \partial_{v}-\gamma_{v}(x)\right] \Phi(x)=Y_{v}(x)
$$

representing wave oscillations along the covector $d x_{v}$ and the vector $d x^{v}$, respectively. The spatial parts of the $d x_{v}$ and $d x^{v}$ are opposite in sign, therefore, multiplets $\Psi(x)$ and $\Phi(x)$ can also be called contra propagating waves. The quantities $\gamma^{v}(x)$ and $\gamma_{v}(x)$ in (1) are defined as the logarithmic derivatives and local wave vectors. The right-hand sides of (1) contain currents, which will be discussed below.

In the absence of commutativity of the multiplication, we can consider differential forms (1) with the reverse order of factors. And in the absence of associativity of the multiplication, the ambiguity of the derivative with three factors arises. It turns out that the multiplicity of values of differential forms (1) corresponds to the hypercomplex dimension of wave motions.

It is obvious that the ambiguity of wave forms (1) passes to the properties of the space metric and generates a plurality of the ray paths of wave motions (branching and consolidation).

\section{Classes of wave motions}

We define an exclusive class of wave forms (1) as generated by exclusive algebras of hypercomplex numbers [1] of dimension $n_{\mathbb{R}}=1,2,4,8$ over the field of real numbers or $n_{\mathbb{C}}=0.5,1,2,4$ over the field of complex numbers. This class of wave motion is generated by doubling of dimensions.

As dimension $\Psi(x)$ and $\Phi(x)\left(n_{\mathbb{R}}\right)$ increase, so does the complexity of wave motions due to an increase in the multiplicity of values of the logarithmic derivatives $\gamma^{v}(x)$ and $\gamma_{v}(x)$ which sets the basis for the local wave vectors. The dimension is growing, and the properties of the algebra of numbers are degrading [1], so when $n_{\mathbb{R}}=4$ multiplying is non-commutative, and when $n_{\mathbb{R}}=8$ not associative (alternative).

Exclusive algebras, judging by the set of their dimensions, determine the structures of only some wave forms. The necessity of extending the exclusive class of wave motions is obvious.

Dimensions $n_{\mathbb{R}}=4$ (a group of motions $\mathrm{SO}(3)$ ) and $n_{\mathbb{R}}=6$ or $n_{\mathbb{C}}=3$ (a group of motions SU(3), which is an octave of $\mathrm{SO}(3))$ begs.

We define an extended class of wave motions as the complement of dimensions 3 and 6.

There are two other dimensions $n_{\mathbb{R}}=5,7$ that were not discussed above. They as well as dimensions $n_{\mathbb{R}}=1,3$ belong to a subclass of odd dimensions, and in designations over a field of complex 
numbers they should be written as $n_{\mathbb{C}}=0.5,1.5,2.5,3.5$, it is a subclass of half integer dimensions, and in contrast to $n_{\mathbb{C}}=1,2,3,4$, it is a subclass of integer dimensions.

The generator of fractal wave motions is obtained if in differential forms (1) multiplicative $\gamma^{\mu}(x)$ and $\gamma_{\mu}(x)$ and additive $Y^{\mu}(x)$ and $Y^{\mu}(x)$ effects are given by random functions. As a result, the wave motions discussed above should be excited.

In this linear mode of fractal oscillation generation, an important role is played by the ratio of dimensions $3: 2$, which generates a diatonic sequence in the dimensions of wave motion, the periodicity of which can be compared to 12 basic fractal dimensions.

It's just a game of dimensions, not a harmonic series. However, if fixed values of the diatonic sequence are assigned to the quantities $\gamma^{\mu}(x)$ and $\gamma_{\mu}(x)$, it will be a random walk through the frequencies of the harmonic series. Obviously, these two types of fractal oscillator as an upset and tuned piano will differ in scaling, which is characterized by a fractional dimension of collective oscillations, a kind of average between the whole and half-whole dimensions. In complex natural systems, this parameter takes values of $0.7-0.9$, which characterize the type of collective oscillations, a mixture of integer and fractal dimensions. This dimension mixing parameter has limit values of 0.5 and 1 , which correspond to the Poisson and lognormal distributions.

\section{Nonlinear equations of Imbedding method}

Systems of nonlinear wave equations are obtained as follows. The waveforms for $\gamma^{\nu}(x)$ and $\gamma_{v}(x)$ are written similarly to (1) using covariant derivatives $D^{v}=i \partial^{v}+\gamma^{v}(x)$ and $D_{v}=i \partial_{v}+\gamma_{v}(x)$, we obtain covariant distortions of the vectors $\gamma^{v}(x)$ and $\gamma_{v}(x)$ :

$$
\begin{gathered}
{\left[i \partial^{v}+\gamma^{v}(x)\right] \gamma^{\mu}(x)=J^{v \mu}(x), \quad\left[i \partial_{v}-\gamma_{v}(x)\right] \gamma_{\mu}(x)=J_{v \mu}(x),} \\
{\left[i \partial^{v}+\gamma^{v}(x)\right] \gamma_{\mu}(x)=J_{\mu}^{v}(x), \quad\left[i \partial_{v}-\gamma_{v}(x)\right] \gamma^{\mu}(x)=J_{v}^{\mu}(x) .}
\end{gathered}
$$

These are the second logarithmic derivatives containing bilinear combinations of $\gamma^{\nu}(x)$ and $\gamma_{\nu}(x)$ (local orts, wave vectors, calibration potentials, etc.), which give the metric and topological tensor, local reflection coefficient, refractive index, impedance, and much more.

The relations (2) are the equations of evolution of local orts tangent to world lines (analogous to the equations of relativistic mechanics), and (3) are analogous to the Riccati equations of the imbedding method [2].

\section{Closing of equations}

We discuss the closing of equations for wave forms. If the values $\gamma^{v}(x)$ and $\gamma_{v}(x)$ are considered as gauge vector potentials, then expressions (2) and (3) are distortion tensors, corresponding to the stresses that can be associated with the deformation (distortion) of the currents $\gamma^{v}(x) \Psi(x)$ and $\gamma_{v}(x) \Phi(x)$ using analogues of Hooke's law.

The second way of closing equations for wave forms. The stress vectors can be obtained from the stress tensors (2) and (3) by means of covariant divergence:

$$
\begin{gathered}
D_{v} D^{v} \gamma^{\mu}(x)=D_{v} J^{v \mu}(x)=T^{\mu}(x), \quad D^{v} D_{v} \gamma_{\mu}(x)=D^{v} J_{v \mu}(x)=T_{\mu}(x), \\
D_{v} D^{v} \gamma_{\mu}(x)=D_{v} J_{\mu}^{v}(x)=T_{\mu}(x), \quad D^{v} D_{v} \gamma^{\mu}(x)=D^{v} J_{v}^{\mu}(x)=T^{\mu}(x)
\end{gathered}
$$


These values can be associated with the current of wave motions $\gamma^{v}(x) \Psi(x)$ and $\gamma_{v}(x) \Phi(x)$, using analogues of Ohm's law:

$$
T^{\mu}(x)=\hat{\rho} \gamma^{\mu}(x) \Psi(x), \quad T_{\mu}(x)=\tilde{\rho} \gamma_{\mu}(x) \Phi(x) .
$$

We obtain a system of nonlinear wave equations (1-6), where the interaction constants (operators) $\hat{\rho}$ and $\tilde{\rho}$ (conductivity) determine the asymmetry of the reflections in the case $\hat{\rho} \neq \tilde{\rho}$.

The third way of closing equations for wave forms is obtained if we once again take the covariant divergence of expressions (4) and (5), as a result we have:

$$
\begin{array}{ll}
D_{\mu} D_{v} D^{v} \gamma^{\mu}(x)=\hat{T}, & D^{\mu} D^{v} D_{v} \gamma_{\mu}(x)=\tilde{T}, \\
D^{\mu} D_{v} D^{v} \gamma_{\mu}(x)=\tilde{T}, & D_{\mu} D^{v} D_{v} \gamma^{\mu}(x)=\hat{T} .
\end{array}
$$

The right parts (7) and (8) can be associated with the $\Psi(x)$ and $\Phi(x)$, the norm of which can be compared to the curvature of space. This method of closing equations for wave forms gives a description of spatial deformations, if $\gamma^{v}(x)$ and $\gamma_{v}(x)$ is understood as displacement vectors.

The fourth order of differential forms in (7) and (8), taking into account the fact that $\gamma^{v}(x)$ and $\gamma_{v}(x)$ are also differential forms, indicates that we are dealing with the theory of deformations, which is essentially the theory of gravity.

Gravity is represented by products of even order of differential forms $\gamma^{v}(x)$ and $\gamma_{v}(x)$ as a theory of transport in statistical Radiophysics [3]. First of all it is bilinear combinations forms and which give the metric tensor and the intensity of the wave motion.

A larger number of co-factors yields higher-order correlations. Asymmetric combinations correspond to the presence of a mean field and can represent all other types of interactions except gravitational. The fourth-order coherence functions, which represent two pairs of waves and give a correlation between the pairs, are responsible for the curvature of space. One pair of waves moves directly in time, and the second pair moves backwards. Scattering back in time can be seen as a dark matter effect. Four-wave motion and large scattering multiplicities can be associated with the Hopf bundle [4, 5]. The four-wave interaction constant must be associated with the gravitational constant. The equations for coherence functions are unclosed [3], we have to consider all the multiplicities of scattering in the motions of matter and sum up the infinite products of wave pairs. This sum is characterized by a logarithmic scaling width $N=\log _{A}(L / l)$, where $L$ and $l$ are external and internal scales.

If we take as the dimensions of the Universe $\left(10^{26} \mathrm{~m}\right)$ and the quark $\left(10^{-19} \mathrm{~m}\right)$, assuming $A=10$ than $N=\log _{A}(L / l)$ will be one of the great numbers [6,7], and $g=1 / N=10^{-45}$ will give in order of magnitude dimensionless gravitational constant. You can renormalize $g$, if instead of the size of the quark to take the size of the nucleon $10^{-15} \mathrm{~m}$, then $g=10^{-41}$.

The fourth method of closing equations for wave forms. Consider the feedback that will give an additive effect of the properties of space on the wave motions (1), in addition to the multiplicative effect.

The fourth method of closing equations for wave forms. We need more feedback, which will give an additive effect of the space properties on the wave motions of (1), while the multiplicative $\gamma^{v}(x)$ and $\gamma_{v}(x)$ in (1) already exist.

If the distortion of the vectors $\gamma^{\nu}(x)$ and $\gamma_{\nu}(x)$, see (2) and (3), which determines the strain and stress, is projected onto the directions $\gamma^{\nu}(x)$ and $\gamma_{\nu}(x)$, and the resulting vector is substituted in the right parts (1) as sources of waves then we obtain feedback that will give a Dynamo effect in wave motions.

Due to this effect, the birth of matter particles is possible in a strong gravitational field. The generation coefficients can be obtained from observations on the renewal of stars on the periphery 
of galaxies. This model can be used to describe the birth of supernovae (explosive events) and the moderate activity of galactic nuclei, accompanied by flows of dark matter and energy (analogous to solar activity, which essentially works as a reversal of the motion of matter). It is clear that this idea will bury the concept of Black holes and give the stars the possibility of rebirth, and on the scales of the Universe, wave motions are possible, in which expansion is only the visible phase, and the invisible compression is simultaneously due to the reverse flows of dark matter and energy, which somehow permeate the entire Cosmos.

\section{Conclusion}

We considered the equation for wave motions. Wave mechanics gives us the rule of how to pass singular points: foci and caustics. This rule should be used when considering Black holes and the Big Bang, and do not forget that it is only an abstraction, and not really existing objects.

To understand what is happening in the center of galaxies, it is necessary to compare their photo with a picture of the center of the Typhoon, where the phase transitions give the kinetic energy necessary to overcome the gravitational attraction. Something similar must take place in the center of galaxies. The prerequisites for this are discussed above. The Big Bang will be replaced by supernova explosions. Oscillations in the fields of matter can explain the local baryon asymmetry, and on the nucleon scales we see the same Hopf bundle as on the scales of galaxies and cyclones, because the basis of the dynamics of the Universe are turbulence and scaling of wave motions.

\section{References}

[1] I.L. Kantor, A.S. Solodovnikov, Hypercomplex numbers (Springer-Verlag, Berlin, New York, 1989)

[2] B.M. Shevtsov, J. Math. Phys., 37, 6130 (1996)

[3] B.M. Shevtsov, J. Math. Phys., 40, 4359 (1999) DOI: 10.1063/1.532972

[4] H. Hopf, Mathematische Annalen, 104, 637-665 (1931) DOI:10.1007/BF01457962

[5] A.R. Shastri, Basic Algebraic Topology (CRC Press, London, New York, 2013)

[6] D.E. Knuth, Science, 194, 1235 (1976) DOI:10.1126/science.194.4271.1235

[7] M. Gardner, Scientific American, 237, 18 (1977) DOI:10.1038/scientificamerican1177-18. 